\title{
COVID-19, HIV-Associated Cryptococcal Meningitis, Disseminated Tuberculosis and Acute Ischaemic Stroke: A Fatal Foursome
}

\author{
Felix Bongomin (I) ${ }^{1,2}$ \\ Senai Goitom Sereke ${ }^{3}$ \\ Jerom Okot $\mathbb{D}^{2}$ \\ Ronald Katsigazi ${ }^{1}$ \\ Tadeo Kiiza Kandole ${ }^{4}$ \\ Anthony Oriekot ${ }^{5}$ \\ Ronald Olum (D) \\ Angella Atukunda ${ }^{6}$ \\ Joseph Baruch Baluku (D) ${ }^{5}$ \\ Frederick Nakwagala ${ }^{6}$ \\ 'Department of Medicine, School of \\ Medicine, Makerere University College of \\ Health Sciences, Kampala, Uganda; \\ ${ }^{2}$ Department of Medical Microbiology \\ and Immunology, Faculty of Medicine, \\ Gulu University, Gulu, Uganda; \\ ${ }^{3}$ Department of Radiology and \\ Radiotherapy, School of Medicine, \\ Makerere University College of Health \\ Sciences, Kampala, Uganda; ${ }^{4}$ Infectious \\ Diseases Institute, Makerere University \\ College of Health Sciences, Kampala, \\ Uganda; ${ }^{5}$ Division of Pulmonology, \\ Kiruddu National Referral Hospital, \\ Kampala, Uganda; ${ }^{6}$ Department of \\ Medicine, Mulago National Referral \\ Hospital, Kampala, Uganda
}

Correspondence: Felix Bongomin Department of Medical Microbiology and Immunology, Faculty of Medicine, Gulu

University, Gulu, Uganda

Tel +256-784-523-395

Email drbongomin@gmail.com
Background: Several viral, bacterial and fungal co-infections have been associated with increased morbidity and mortality among patients with COVID-19. We report a fatal case of severe COVID-19 pneumonia in a patient with a recent diagnosis of advanced HIV disease complicated by cryptococcal meningitis, disseminated tuberculosis and acute ischemic stroke.

Case Presentation: A 37-year-old Ugandan woman was diagnosed with HIV infection 8 days prior to her referral to our center. She was antiretroviral naïve. Her chief complaints were worsening cough, difficulty in breathing, fever and altered mental status for 3 days with a background of a 1-month history of coughing with associated drenching night sweats and weight loss. The reverse transcriptase-polymerase chain reaction for SARS-CoV-2 of her nasopharyngeal swab sample was positive. Chest radiograph demonstrated military pattern involvement of both lungs. The serum and cerebrospinal fluid cryptococcal antigen tests were positive. Urine lipoarabinomannan and sputum GeneXpert were positive for Mycobacterium tuberculosis. Computed tomography of the brain showed a large acute ischemic infarct in the territory of the right middle cerebral artery. Regardless of the initiation of treatment, that is, fluconazole $1200 \mathrm{mg}$ once daily, enoxaparin $60 \mathrm{mg}$, intravenous (IV) dexamethasone $6 \mathrm{mg}$ once daily, oral fluconazole $1200 \mathrm{mg}$ once daily, IV piperacillin/tazobactam $4.5 \mathrm{~g}$ three times daily and oxygen therapy, the patient passed on within 36 hours of admission.

Conclusion: Co-infections worsen COVID-19 outcomes.

Keywords: COVID-19, HIV, cryptococcal meningitis, stroke, tuberculosis, co-infection

\section{Background}

Respiratory fungal and bacterial infections occur as co-infections or a complication (superinfection) of the coronavirus disease - 2019 (COVID-19). ${ }^{1-3}$ At least $19 \%$ and $24 \%$ of people with COVID-19 have a co-infection or superinfection, respectively, which increase the risk of mortality by three-fold. ${ }^{4}$ Fungal co-infections and bacterial-fungal co-infections are frequently seen in patients with severe COVID19 , and are associated with substantial co-morbidity and mortality. ${ }^{3}$

In sub-Saharan Africa, tuberculosis (TB) and cryptococcal meningitis (CM) are the most common cause of HIV-related deaths. ${ }^{5,6}$ In Uganda, in addition to TB and $\mathrm{CM}$ being the most common cause of morbidity and mortality among persons with advanced HIV disease, ${ }^{7}$ up to $14 \%$ of patients with $\mathrm{CM}$ have active TB with an associated increased mortality among TB-CM co-infected individuals. ${ }^{8}$

Reports on triple co-infection of HIV, severe acute respiratory syndrome coronavirus-2 (SARS-CoV-2) and Mycobacterium tuberculosis are few and 
current reports do not suggest that the triple infection worsens outcomes. ${ }^{9}$ Herein, we describe a fatal case of COVID-19 pneumonia in a patient with HIV-associated cryptococcal meningitis, ischemic stroke and disseminated tuberculosis. In addition, we searched PubMed and Google scholar to identify additional cases of fungal and tuberculosis co-infections in patients with COVID-19.

\section{Case Presentation}

A 37-year-old nulliparous woman with a past medical history of a recent diagnosis of HIV infection not on antiretroviral therapy was admitted at the Mulago National Referral Hospital (MNRH) COVID-19 Treatment Unit (CTU), Uganda with a 3-day history of worsening cough and difficulty in breathing. She had had a productive cough with mucoid sputum accompanied by evening fevers and weight loss of a 2-month duration. On the referral note, there was also a documented history of anosmia, high-grade intermittent fevers, headaches, altered mentation, an episode of loss of consciousness and myalgias for a duration of 5 days. Four days prior to referral to MNRH CTU from a general hospital, her nasopharyngeal swab for SARS-CoV-2 was positive on both reverse transcriptase-polymerase chain reaction (RT-PCR) and rapid antigen tests. She had tested positive for HIV infection 8 days prior to admission and was not undergoing antiretroviral therapy or cotrimoxazole prophylaxis.

On presentation (day 0 ), she was critically ill, febrile with an axillary temperature of $38.5^{\circ} \mathrm{C}$, a tachycardia of 168 beats per minute, tachypnoea of 32 breaths per minute, random blood sugar of $8.7 \mathrm{mmol} / \mathrm{L}$, blood pressure of $132 / 76 \mathrm{mmHg}$ and was hypoxic with a saturation ranging between $85-90 \%$ on 5 liters per minute via a nasal prong. Respiratory examination revealed nasal flaring, with intercostal recessions. There were widespread, bilateral, coarse crepitations. She was restless with a Glasgow coma scale of 8/15 (eye opening 3 , best verbal response 2 and best motor response 3 ). Her neck was stiff, with a positive Kernig's sign. Other systems were unremarkable.

Her serum and cerebrospinal fluid cryptococcal antigen (CrAg) (Immy Inc., Norman, OK) tests were positive (Figure 1). A blood smear was negative for Plasmodium and other haemoparasites. Complete blood count revealed a white blood cell count of $11.8 \times 10^{3} / \mu \mathrm{L}$, with a neutrophilia of $10.8(91.6 \%)$ and a lymphopenia of $0.53(4.5 \%)$. She had a microcytic anemia with

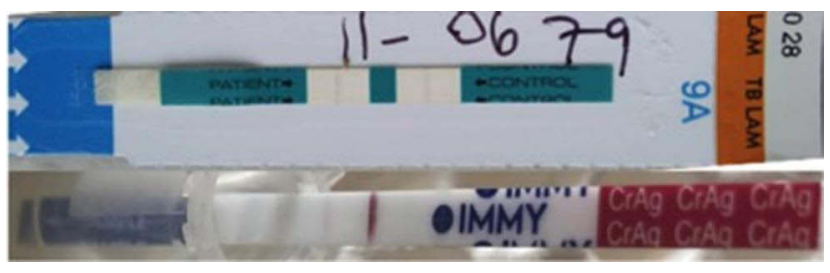

Figure I LAM TB (upper) and cryptococcal antigen test (lower) results.

hemoglobin level of $8.5 \mathrm{~g} / \mathrm{dl}$ and mean corpuscular volume of $67 \mathrm{fL}$. Her platelet levels were normal at $300 \times 10^{3} / \mu \mathrm{L}$. Renal and liver biochemistry were within limits. Her blood group was B rhesus D positive. The urine lipoarabinomannan (LAM) was positive (Grade II) (Figure 1). Chest radiography revealed features of miliary tuberculosis with background ground glass opacities (Figure 2). Unenhanced computed tomography of the brain demonstrated a right sided large geographic area of hypodensity (19-25 HU) involving the right frontoparietotemporal cortex in the territory of the right middle cerebral artery with an associated hyperdense middle cerebral artery sign (Figure 3A and B). Sputum and blood samples were taken off for GeneXpert and cultures, respectively. The GeneXpert was positive without rifampicin resistance. Anaerobic, aerobic, fungal and mycobacterial cultures were negative. CD4+ T-cell count was 32 cell/microliter and HIV RNA viral load was unknown. Her urine human chorionic gonadotrophin was negative.

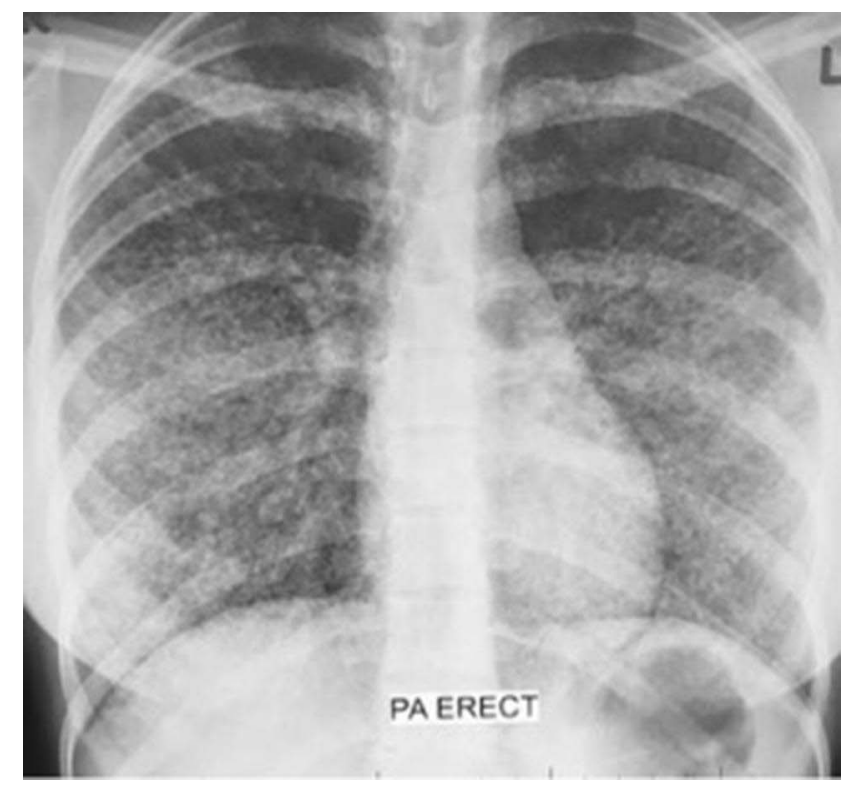

Figure 2 Anteroposterior chest radiography demonstrated diffuse reticulonodular opacities (nodular> reticular) involving the bilateral hemithorax with background ground glass haziness. 


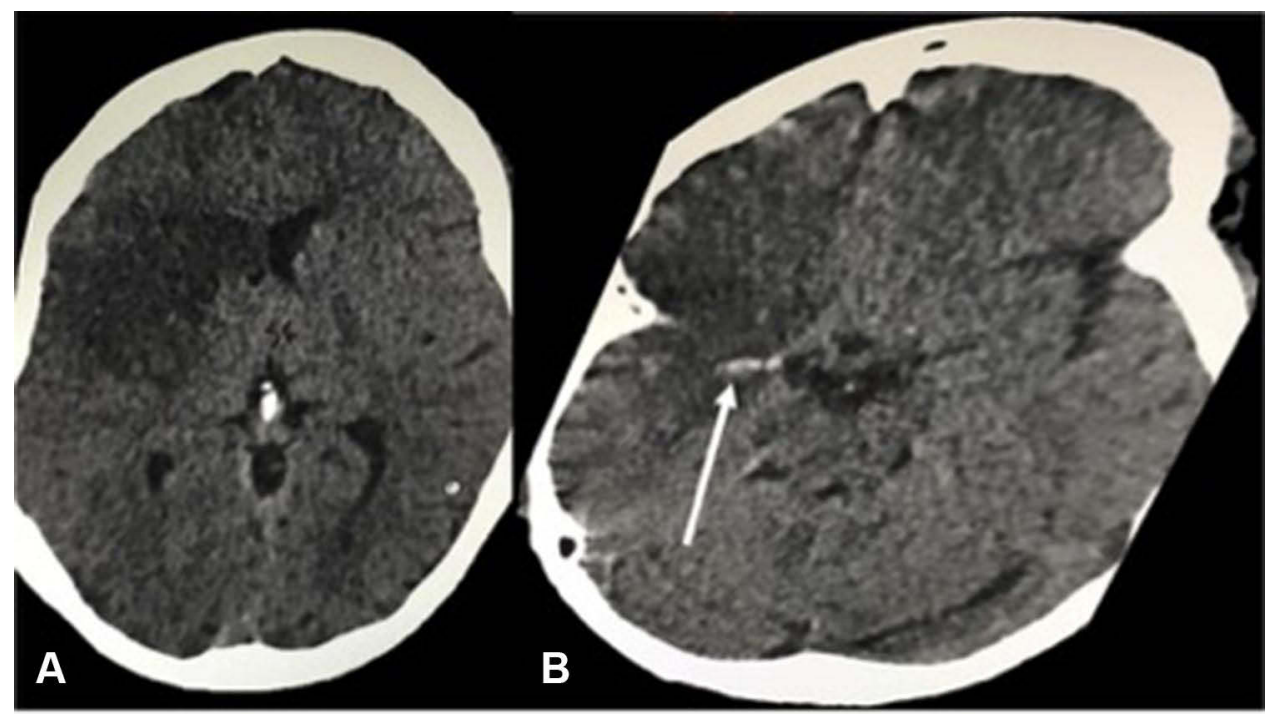

Figure 3 Unenhanced computed tomography of brain, (A) axial image at the level of anterior horns of the lateral ventricles demonstrating a large geographic area of hypodensity involving the right frontopareitotemporal cortex. (B) axial image at the level of circle of Wills demonstrating hyperdense middle cerebral artery sign (white arrow) of the $\mathrm{MI}$ segment of the right middle cerebral artery.

A diagnosis of severe COVID-19 in the background of advanced HIV infection with complications of CM, disseminated tuberculosis and microcytic anemia was made. She was initiated on intravenous dexamethasone at a dose of $6 \mathrm{mg}$ once daily, subcutaneous enoxaparin $60 \mathrm{mg}$ once daily, intravenous paracetamol $1 \mathrm{~g}$ three times a day, cotrimoxazole $960 \mathrm{mg}$ once daily, oral fluconazole $1200 \mathrm{mg}$ once daily and intravenous piperacillin/tazobactam 4.5 $\mathrm{g}$ three times daily. Also, $300 \mathrm{mg}$ of aspirin was administered as a STAT dose followed by $75 \mathrm{mg}$ once daily. AntiTB drugs were not available at the CTU and were being sought from the TB treatment unit. On day +1 , a nasogastric tube was passed for feeding and urethral catheter was passed to monitor urine output.

Despite an escalation of oxygen delivery via nonrebreather mask (at $15 \mathrm{~L}$ per minute), and later high-flow nasal cannula (at 50 liters per minute), the patient continued to deteriorate with an increasing oxygen requirement and worsening hypoxemia. She passed on within 36 hours of hospitalization, as we were looking for intensive care unit space for assisted ventilation.

\section{Discussion}

The burden of AIDS-defining illnesses, particularly TB and $\mathrm{CM}$ are high in sub-Saharan African countries with an estimated rate ranging from $16 \%$ to $29 \% .{ }^{10}$ Our patient had evidence of both $\mathrm{CM}$ and disseminated TB, which are the two most common causes of AIDS-related deaths in sub-Saharan Africa. The most unusual presentation in this patient was the occurrence of these two life-threatening opportunistic infections in association with two other equally lethal conditions - severe COVID-19 pneumonia and acute ischemic stroke. These explain the fatality observed in this patient confronted with a quartet of lifethreatening conditions. The ischemic stroke could also be a sequel of CM, SARS-CoV-2 or tuberculous meningitis. In a large cohort of southern Africans with COVID-19, 9.1\% had HIV and 3.6\% had tuberculosis. ${ }^{11}$ In this cohort, HIV patients not on anti-retroviral therapy were more likely to die. This observation has also been reported in a patient from Ethiopia who had a COVID-19-HIV-tuberculosis triple infection. ${ }^{12}$ The patient was on antiretroviral therapy for over three years and survived COVID-19 after hospitalization for over 10 days.

Since COVID-19 hit the world in December 2019, several reports on respiratory co-infection with different bacterial (including TB), viral (influenza and other viruses) and fungal (Cryptococcus, Histoplasma, Aspergillus and others) infections have been reported. ${ }^{3}$ The prevalence of TB has been reported to be higher in patients with severe COVID-19 than those with non-severe disease. A study from China reported on overall prevalence to range between 0.47 to $4.47 \%{ }^{13}$ Results of a cohort study from European countries showed that COVID-19 can present simultaneously, before or after the diagnosis of TB. However, the cohort also left some unanswered question regarding the role of COVID-19 in TB re-activation, the role of TB in COVID-19 pathogenesis and outcomes of 
co-infections. ${ }^{14,15}$ Our patient likely had pre-existing, undiagnosed TB, given her 1-month history of cough and the associated B-symptoms. TB is associated with extensive parenchymal lung damage. This probably explains the rapid progression of the respiratory symptoms in our patient.

On the other hand, there are few reports of COVID-19 and fungal co-infection in the literature. ${ }^{16}$ In China, fungal co-infection was reported in up to about $6 \%$ of patients with COVID-19. ${ }^{17,18}$ The most common fungal etiologies associated with COVID-19 in the above studies were C. albicans, A. flavus, and A. fumigatus. ${ }^{16,19}$ There have been two recent cases of COVID-19-CM co-infections. ${ }^{20}$ $\mathrm{HIV}$-associated CM is the most common cause of meningitis, accounting for 15-20\% of AIDS-related deaths globally and Cryptococcus is the most common etiology of adult meningitis in sub-Saharan Africa. ${ }^{21}$ Clinically, our patient had advanced HIV disease which was immunologically confirmed by the low CD4+ T-cell count.

The relationship between COVID-19 infection and cerebrovascular disease is poorly understood. ${ }^{22}$ However, previous studies have demonstrated an association between an increased severity and mortality of COVID-19 with a previous history of stroke. ${ }^{23}$ In a recent systematic review and meta-analysis of over 100,000 patients with COVID-19, 1.4\% developed a cerebrovascular accident in which $87.4 \%$ were acute ischemic stroke and the rest hemorrhagic stroke. ${ }^{22}$ The most important predictors of stroke in these patients were advanced age, pre-existing vascular risk factors and severe COVID-19 illness. $^{22}$ However, compared with patients without COVID-19, COVID-19 patients who developed stroke were younger and suffered large vessel occlusion. ${ }^{22}$ Consistent with the above metaanalysis, our patient was younger and sustained right middle cerebral artery occlusion. This case report also highlights the uncertainty surrounding the risk and management of ischemic stroke in patients with COVID-19 pneumonia who are already receiving anticoagulant therapy. $^{24}$

The concurrent management of disseminated TB, CM, COVID-19 and cerebrovascular disease in a patient immunocompromised by HIV is expectedly complex with no standardized guidelines. The potential for drug-drug interaction and adverse events is anticipated in this scenario. For example, in this patient, rifampicin would induce the cytochrome systems significantly reducing plasma levels of fluconazole.

\section{Conclusion}

COVID-19, disseminated TB, CM and acute ischemic stroke quadruple co-morbidities in the background of HIV infection have been reported previously. Disease progression was rapid with fatal consequences. This case reminds clinicians to always suspect and investigate for additional opportunistic infections in the presence of one in the setting of advanced HIV disease.

\section{Data Sharing Statement}

The information used and/or analyzed during this case report is available from the corresponding author on reasonable request.

\section{Ethics Approval and Consent to Participate}

Ethical approval was sought from Mulago Hospital Research and Ethics Committee (Reference number MHREC 2030). Consent to publish this case report was obtained from the next of kin of the deceased patient.

\section{Acknowledgments}

We would like to acknowledge, the patient and her caretakers, Mulago National Referral Hospital and its staff for they actively supported the process of data collection and patient follow-ups.

\section{Author Contributions}

All authors made a significant contribution to the work reported, whether that is in the conception, study design, execution, acquisition of data, analysis and interpretation, or in all these areas; took part in drafting, revising or critically reviewing the article; gave final approval of the version to be published; have agreed on the journal to which the article has been submitted; and agree to be accountable for all aspects of the work.

\section{Disclosure}

The authors declare that they have no conflicts of interest in this work.

\section{References}

1. Alhumaid S, Al Mutair A, Al Alawi Z, et al. Coinfections with bacteria, fungi, and respiratory viruses in patients with SARS-CoV-2: a systematic review and meta-analysis. Pathogens. 2021;10(7):809. doi:10.3390/pathogens10070809

2. Lansbury L, Lim B, Baskaran V, Lim WS. Co-infections in people with COVID-19: a systematic review and meta-analysis. $J$ Infect. 2020;81(2):266-275. doi:10.1016/j.jinf.2020.05.046 
3. Zhu X, Ge Y, Wu T, et al. Co-infection with respiratory pathogens among COVID-2019 cases. Virus Res. 2020;285:198005. doi:10.1016/j.virusres.2020.198005

4. Musuuza JS, Watson L, Parmasad V, Putman-Buehler N, Christensen L, Safdar N. Prevalence and outcomes of co-infection and superinfection with SARS-CoV-2 and other pathogens: a systematic review and meta-analysis. PLoS One. 2021;16(5): e0251170. doi:10.1371/journal.pone.0251170

5. Ford N, Meintjes G, Calmy A, et al. Managing advanced HIV disease in a public health approach. Clin Infect Dis. 2018;66(Suppl 2):S106SS110. doi:10.1093/cid/cix1139

6. Osler M, Hilderbrand K, Goemaere E, et al. The continuing burden of advanced HIV disease over 10 years of increasing antiretroviral therapy coverage in South Africa. Clin Infect Dis. 2018;66(Suppl 2):S118-S125. doi:10.1093/cid/cix1140

7. Ellis J, Bangdiwala AS, Cresswell FV, et al. The changing epidemiology of HIV-Associated adult meningitis, Uganda 2015-2017. Open Forum Infect Dis. 2019;6(10):ofz419. doi:10.1093/ofid/ofz419

8. Rutakingirwa MK, Cresswell FV, Kwizera R, et al. Tuberculosis in HIV-associated cryptococcal meningitis is associated with an increased risk of death. J Clin Med. 2020;9(3):781. doi:10.3390/ jem9030781

9. González-Domenech CM, Pérez-Hernández I, Gómez-Ayerbe C, Viciana Ramos I, Palacios-Muñoz R, Santos J. A pandemic within other pandemics. when a multiple infection of a host occurs: SARS-CoV-2, HIV and Mycobacterium tuberculosis. Viruses. 2021;13(5):931. doi:10.3390/v13050931

10. Reinhardt SW, Spec A, Meléndez J, et al. AIDS-defining illnesses at initial diagnosis of HIV in a large Guatemalan cohort. Open Forum Infect Dis. 2017;4(4):ofx249. doi:10.1093/ofid/ofx249

11. Jassat W, Cohen C, Tempia S, et al. Risk factors for COVID-19related in-hospital mortality in a high HIV and tuberculosis prevalence setting in South Africa: a cohort study. Lancet HIV. 2021;8(9): e554-e567.

12. Tolossa $T$, Tsegaye R, Shiferaw $\mathrm{S}$, et al. Survival from a triple co-infection of COVID-19, HIV, and tuberculosis: a case report. Int Med Case Rep J. 2021;14:611-615. doi:10.2147/IMCRJ.S326383

13. Gao Y, Liu M, Chen Y, Shi S, Geng J, Tian J. Association between tuberculosis and COVID-19 severity and mortality: a rapid systematic review and meta-analysis. J Med Virol. 2021;93(1):194-196. doi: $10.1002 / j m v .26311$
14. Visca D, Ong CWM, Tiberi S, et al. Tuberculosis and COVID-19 interaction: a review of biological, clinical and public health effects. Pulmonology. 2021;27(2):151-165. doi:10.1016/j.pulmoe.2020.12.012

15. Tadolini M, Codecasa LR, García-García JM, et al. Active tuberculosis, sequelae and COVID-19 co-infection: first cohort of 49 cases. Eur Respir J. 2020;56(1):2001398. doi:10.1183/13993003.013982020

16. Song G, Liang G, Liu W. Fungal co-infections associated with global COVID-19 pandemic: a clinical and diagnostic perspective from China. Mycopathologia. 2020;185;599-606.

17. Chen N, Zhou M, Dong X, et al. Epidemiological and clinical characteristics of 99 cases of 2019 novel coronavirus pneumonia in Wuhan, China: a descriptive study. Lancet. 2020;395 (10223):507-513. doi:10.1016/S0140-6736(20)30211-7

18. Yang X, Yu Y, Xu J, et al. Clinical course and outcomes of critically ill patients with SARS-CoV-2 pneumonia in Wuhan, China: a single-centered, retrospective, observational study. Lancet Respir Med. 2020;8(5):475-481. doi:10.1016/S2213-2600(20)30079-5

19. Koehler P, Cornely OA, Böttiger BW, et al. COVID-19 associated pulmonary aspergillosis. Mycoses. 2020;63(6):528-534. doi:10.1111/ myc. 13096

20. Ghanem H, Sivasubramanian G. Cryptococcus neoformans meningoencephalitis in an immunocompetent patient after COVID-19 infection. Case Rep Infect Dis. 2021;2021:e5597473.

21. Rajasingham R, Smith RM, Park BJ, et al. Global burden of disease of HIV-associated cryptococcal meningitis: an updated analysis. Lancet Infect Dis. 2017;17(8):873-881. doi:10.1016/S14733099(17)30243-8

22. Nannoni S, de Groot R, Bell S, Markus HS. Stroke in COVID-19: a systematic review and meta-analysis. Int $J$ Stroke. 2021;16 (2):137-149. doi:10.1177/1747493020972922

23. Aggarwal G, Lippi G, Michael Henry B. Cerebrovascular disease is associated with an increased disease severity in patients with Coronavirus Disease 2019 (COVID-19): a pooled analysis of published literature. Int J Stroke. 2020;15(4):385-389. doi:10.1177/ 1747493020921664

24. Zakeri A, Jadhav AP, Sullenger BA, Nimjee SM. Ischemic stroke in COVID-19-positive patients: an overview of SARS-CoV-2 and thrombotic mechanisms for the neurointerventionalist. J Neurointerventional Surg. 2021;13(3):202-206. doi:10.1136/neurintsurg-2020-016794
Infection and Drug Resistance

\section{Publish your work in this journal}

Infection and Drug Resistance is an international, peer-reviewed openaccess journal that focuses on the optimal treatment of infection (bacterial, fungal and viral) and the development and institution of preventive strategies to minimize the development and spread of resistance. The journal is specifically concerned with the epidemiology of antibiotic resistance and the mechanisms of resistance development and diffusion in both hospitals and the community. The manuscript management system is completely online and includes a very quick and fair peerreview system, which is all easy to use. Visit http://www.dovepress.com/ testimonials.php to read real quotes from published authors. 